\title{
The role of adhesion molecules in nephropathies: The diagnostic applications
}

\author{
Samuel N Uwaezuoke* \\ Pediatric Nephrology Firm, Department of Pediatrics, University of Nigeria Teaching Hospital, Ituku-Ozalla, Enugu Nigeria
}

\begin{abstract}
Adhesion molecules play a critical role in the pathogenesis of several forms of nephropathies. In this review, their role in renal diseases (especially nephropathies) and their diagnostic applications as biomarkers are discussed. Adhesion molecules which belong to the chemokine's family (interleukin-18 and RANTES), and the immunoglobulin superfamily (intercellular adhesion molecule-1 and vascular cell adhesion molecule-1) are reportedly elevated in diabetic nephropathy, and can thus serve as predictors of the disease. Both intercellular adhesion molecule-1 (ICAM-1) and vascular cell adhesion molecule-1 (VCAM-1) are also highly expressed in other nephropathies, such as proliferative grades of lupus nephritis, Ig A nephropathy, gouty nephropathy, membranous nephropathy, and minimal change nephropathy. However, there is still poor documentation on the role of other adhesion molecules like intercellular adhesion molecule-2 (ICAM-2) and P-selectin in nephropathies despite recent reports about the utility of E-selectin and P-selectin as predictive biomarkers of diabetic nephropathy
\end{abstract}

\section{Introduction}

Adhesion molecules refer to a diverse group of ligands/receptor molecules which facilitate cell adhesion either to other cells or to the extracellular matrix [1]. They are classified into three major groups based on molecular, structural as well as functional differences, and these consist of the integrins, the selectins and those included in the immunoglobulin superfamily. However, chemokines which act as ligands that bind to specific endothelial or extracellular-matrix receptors have been described and regarded as adhesion molecules as well [2].

In health and disease, cell adhesion is vital in the following processes: the recirculation and movement of leukocytes [3], cell differentiation and tissue organization for maintenance of tissue architecture [4], the activation of and communication between immune cells [5], as well as the multiplication and metastatic spread of neoplastic cells [6].

Specifically, leukocyte adhesion is a fundamental event in inflammation, as leukocytes have been reported as vital mediators of injury in several types of glomerulonephritis, vasculitis, tubulointerstitial nephritis and allograft rejection $[7,8]$. Furthermore, changes in levels of adhesion molecules and cytokines have been demonstrated in diabetic nephropathy, before and after dialysis: a finding which underscores their diagnostic role in this renal microvascular complication [9].

In this review, the role of adhesion molecules in renal diseases (especially nephropathies) and their diagnostic applications as biomarkers are discussed.

\section{The major groups of adhesion molecules}

The major families of adhesion molecules as previously mentioned comprise the integrins, the selectins, the immunoglobulin superfamily and the chemokines (chemotactic cytokines) (Figure 1). These

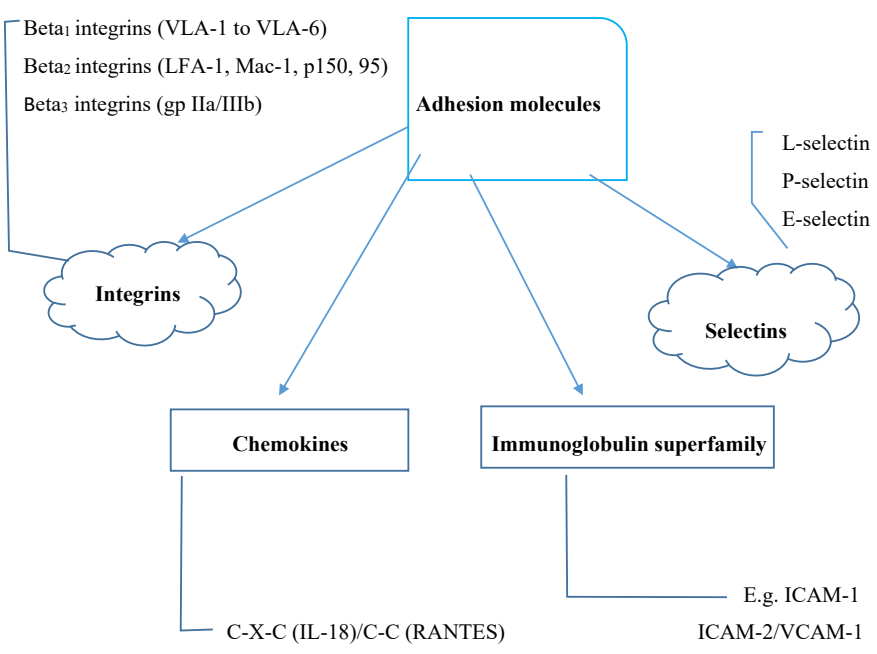

Figure 1. The major families of adhesion molecules and their subfamily members VLA: Very late antigen; IL-18: Interleukin-18; RANTES: Regulated on activation, normal $\mathrm{T}$ cell expressed and secreted; ICAM: Intercellular adhesion molecule; VCAM: Vascular cell adhesion molecule

molecules are expressed on leukocytes, epithelium, endothelium, and some non-vascular cell types. Interestingly, they are also expressed on resident renal cells. Their expression on these latter cells largely forms the basis for their role in nephropathies.

${ }^{\star}$ Correspondence to: SN Uwaezuoke, Department of Pediatrics, UNTH ItukuOzalla, Enugu Postal Code 400001 Nigeria, Tel: +234 8033248108; E-mail: snuwaezuoke@yahoo.com

Key words: adhesion molecules, biomarkers, interleukin-18, rantes, intercellular adhesion molecule-1, vascular cell adhesion molecule-1, nephropathies

Received: February 20, 2019; Accepted: March 06, 2019; Published: March 11, 2019 


\section{Integrins}

Integrins are basically mediators of intercellular and cellmatrix adhesions. The biochemical structure of integrins consists of heterodimeric glycoproteins located on cell membranes with two subunits: a larger alpha chain and a smaller beta chain [6]. Their classification is predicated on the structure of their beta subunits. Thus, the following three subfamilies are recognized: beta-1 integrins or very late antigens (VLA), beta-2 integrins or leukocyte integrins, and beta-3 integrins or cytoadhesins $[5,10,11]$.

The beta-1 integrins are the products of the combination of the beta- 1 chain with different alpha chains and are numbered VLA-1 to VLA- 6 depending on the alpha chain number $\left(\alpha_{1}\right.$ to $\left.\alpha_{6}\right)$. These integrins are expressed on leukocytes, platelets, fibroblasts, epithelial cells, and endothelial cells, and characteristically attach to extra-cellular matrix proteins especially, laminin, collagen, and fibronectin [1]. Therefore, VLAs mediate the attachment of endothelial and epithelial cells to underlying layers [12], adhesion of platelets to the exposed subendothelial matrix, tissue repair, and localization of leukocytes at sites of inflammation [6]. Nevertheless, VLA-4 which is expressed on lymphocytes, monocytes, basophils, and eosinophils plays the role of a receptor for both fibronectin and vascular cell adhesion molecule-1 (VCAM-1): a ligand of the immunoglobulin superfamily expressed on endothelial cells. VLA-4 thus facilitates the interactions between leukocytes and endothelial cells.

The beta- 2 integrins comprise three members which are specifically expressed on leukocytes, namely LFA-1, Mac-I and p150, 95. Whereas LFA-1 is found on almost all immunocytes, Mac-1 and p150, 95 are mainly expressed on monocytes and granulocytes [1]. Given the recognition of beta-2 integrins with varied pseudonyms, the use of cluster designation (CD) nomenclature for purposes of uniformity has been advocated. Thus, these molecules are also known as CD 11a/ CD18, CD 11b/CD18, and CD11c/CD18; CD11 and CD18 referring to $\alpha$ and $\beta$ subunits respectively [13].

The beta- 3 integrins are expressed on the following cells: platelets, endothelium, polymorphonuclear cells, and monocytes. While all attach to fibrinogen and von Willebrand factor (VWF), some also attach to fibronectin and vitronectin. A member of beta-3 integrins, known as gp IIb/IIIa, is the main integrin of platelets where it cleaves to soluble fibrinogen and VWF [1].

\section{Selectins}

The selectins refer to three surface glycoproteins with a similar structure comprising lectin and epidermal growth factor (EGF) domains, as well as complement binding-like modules [14]. The nomenclatureselectin- reflects the functional relevance of the lectin domain and the selectivity of distribution and function of these molecules [1]. The lectin and EGF domains are important for mediating adhesion while the complement binding-like modules provide the mechanical support which ensures that the lectin and EGF domains are kept away from the cell surface. The nomenclature of the selectins is based on the cell type on which they were initially identified: E-selectin (endothelial cells), P-selectin (platelets) and L-selectin (leukocytes). Concerning their sites of expression, E-selectin is exclusively expressed on endothelial cells, P-selectin on both platelets and endothelial cells, while L-selectin is expressed on lymphocytes, neutrophils and monocytes [15-17]. These three adhesion molecules attach to one or more carbohydrate ligands [18].

\section{Immunoglobulin superfamily}

The adhesion molecules which belong to this group are essentially ligands of integrin receptors. Representative members of the group include ICAM-1, ICAM-2 (intercellular adhesion molecule-I and -2), and VCAM-1. Both ICAM-1 and ICAM-2 are expressed on endothelial cells, but the expression of the latter is not modified by inflammatory stimuli. In contrast, expression of ICAM-1 on endothelial cells is increased several folds following cell activation with IL- $1 \beta$, TNF- $\alpha$ or $\gamma$-interferon: which are biomarkers of inflammation [19]. Besides endothelial cells, other cell types such as leukocytes, epithelial cells, dendritic cells, and fibroblasts also express ICAM-1 post-exposure to inflammatory stimuli. Lastly, VCAM-1 is expressed on endothelial cells and several non-vascular cell types such as dendritic cells in lymph node and skin, bone marrow stromal cells and synovial cells [14].

\section{Chemokines}

The chemokines (chemotactic cytokines) constitute a superfamily of polypeptides with the distinctive presence of four retained cysteine (C) residues. Two subfamilies are identified based on the location of another amino acid $(\mathrm{X})$ in between the first two $\mathrm{C}$ residues (alpha or $\mathrm{C}$-X-C subfamily), or the adjacent location of these two $\mathrm{C}$ residues (beta or C-C subfamily) [1]. Representative members of the two subfamilies are interleukin-18 (IL-18) [C-X-C type] and regulated on activation, normal $\mathbf{T}$ cell expressed and secreted (RANTES) [C-C type]; both of them are involved in inflammation by their participation in leukocyte recruitment. These chemokines function through a mechanism akin to adhesion molecules than to classic chemotactic factors. Their cellular distribution determines the responding cell; thus, the IL-18 receptor is confined to neutrophils, whereas the RANTES or CCL-5 receptor is expressed on basophils, eosinophils, monocytes, and a subset of $\mathrm{T}$ lymphocytes [20].

\section{Adhesion molecules and nephropathies}

Interestingly, adhesion molecules are also expressed on native renal cells. A review of the expression of adhesion molecules in normal renal tissue is key to understanding their pathogenic role in nephropathies. For instance, the expression of integrins on different parts of the renal tissue varies, depending on the members of the integrin subfamilies (Table 1). Among the beta-1 integrins, VLA-1 is expressed on mesangium and Bowman's capsule, and on all tubular cells; VLA-2 is expressed on endothelium and distal tubule; VLA3 on mesangium, visceral and parietal epithelia, endothelium and distal tubule; VLA-5 on mesangium and endothelium while VLA- 6 is expressed on all tubular cells [1]. One of the beta-2 integrins, LFA-1, is expressed on monocytes/macrophages located at the glomeruli, as well as the interstitium and vessels. In the immunoglobulin superfamily, ICAM-1 is expressed on glomerular and vascular endothelia while VCAM-1 is expressed on glomerular parietal epithelium and the proximal tubule. Among the selectins, E-selectin is expressed on peritubular capillaries.

Leukocyte adhesion promotes a dynamic relationship between phagocytes and renal parenchymal cells [13]. This ultimately plays a role in the pathophysiology of renal inflammation. Leukocyte adhesion possibly contributes to glomerular cell damage and alteration of the filtration barrier by promoting the release of reactive oxygen species and proteases which are contiguous to glomerular cells and the glomerular basement membrane. In addition, leukocyte adhesion may function as amplifiers of the levels and spectrum of lipid mediators at 
Table 1. Expression of adhesion molecules in normal renal tissue

\begin{tabular}{|c|c|}
\hline Adhesion molecules & Sites of expression in renal tissue \\
\hline \multicolumn{2}{|l|}{ Beta-1 integrins } \\
\hline -VLA-1 & $\begin{array}{l}\text {-Glomerular mesangium, Bowman's capsules, and all } \\
\text { tubular cells }\end{array}$ \\
\hline -VLA-2 & -Glomerular endothelium and distal tubule \\
\hline -VLA-3 & $\begin{array}{l}\text {-Glomerular mesangium, visceral and parietal } \\
\text { epithelia, endothelium and distal tubule }\end{array}$ \\
\hline -VLA-5 & -Glomerular mesangium and endothelium \\
\hline -VLA-6 & -All tubules \\
\hline \multicolumn{2}{|l|}{ Beta-2 integrins } \\
\hline -LFA-1 & -Glomerular monocytes and macrophages \\
\hline \multicolumn{2}{|l|}{ Immunoglobulin superfamily } \\
\hline -ICAM-1 & -Glomerular endothelium and vascular endothelium \\
\hline -VCAM-1 & -Glomerular parietal epithelium and proximal tubule \\
\hline \multicolumn{2}{|l|}{ Selectins } \\
\hline -E-selectin & -Peritubular capillaries \\
\hline
\end{tabular}

VLA: Very late antigen; ICAM-1: Intercellular adhesion molecule-1; VCAM-1: Vascular cell adhesion molecule- 1

inflammation sites via the production of eicosanoids by transcellular routes. Specifically, adhesion may prime phagocyte 5-lipoxygenase pathways, thereby promoting the phagocytic generation and release of leukotriene A4 $\left(\mathrm{LTA}_{4}\right)$ [21].

Firstly, increased expression of ICAM-1 occurs in the glomeruli, proximal tubular cells, and interstitial cells in several inflammatory kidney diseases such as active crescentic glomerulonephritis, mesangioproliferative glomerulonephritis, Ig A nephropathy, HenochSchönlein vasculitis, and proliferative grades of lupus nephritis (class II and IV) [22-27]. Specifically, in advanced sclerotic disease, glomerular ICAM-1 levels may be reduced. However, ICAM-1 levels may either be normal or reduced in minimal change nephropathy [23], whereas one report suggests that circulating levels of ICAM-1 are elevated in some forms of glomerulonephritis [28].

Secondly, in another study which evaluated VCAM-1 expression in renal biopsy specimens in patients with a variety of glomerulonephritides and tubulointerstitial diseases, this adhesion molecule was found to be natively expressed on renal tubular cells in those patients with vasculitis and crescentic nephritis, lupus nephritis (class II, III, IV, and V), IgA nephropathy, and acute interstitial nephritis induced by non-steroidal anti-inflammatory drugs (NSAIDs) [29]. In fact, results from animal experimental studies indicate that enhanced ICAM-1 and VCAM-1 expression confers increased adhesiveness on the renal parenchyma in lupus nephritis and serves as a pathway by which inflammatory cells adhere to renal tissue and mediate renal damage [30,31]. Interestingly, VCAM-1 levels were highest in patients who had vasculitis, with a weak positive correlation between the level of expression and the intensity of leukocytic infiltration. There appears to be a variable pattern in VCAM-l expression, as it was not observed on vascular endothelial cells in any disease, despite the presence of a severe infiltrate. However, prominent expression of VCAM-1 was also noted in proximal tubules of patients with diabetic nephropathy, gouty nephropathy, minimal change nephropathy, and membranous nephropathy: diseases not usually characterized by renal leukocytic infiltration.

Thirdly, concerning the role of selectins in nephropathies, the expression of E-selectin is poorly established although an earlier report suggests its native expression on glomerular endothelial cells in some patients with acute glomerulonephritis, lupus nephritis, and IgA nephropathy, but not in those with focal segmental glomerulosclerosis or membranous nephropathy [32].

In more recent studies, the serum levels of ICAM-1, VCAM-1, E-selectin, and P-selectin were demonstrated to be elevated in diabetic nephropathy $[9,33]$. There is indeed a strong correlation between adhesion molecules and diabetic nephropathy. Adhesion molecules are reportedly elevated in patients with diabetes mellitus [34]. In the pathogenic trajectory of diabetic nephropathy, the changes in renal architecture triggered by hyperglycemic injury are linked to several cellular events and activation of signaling pathways [35]. The main pathogenic components of diabetic nephropathy include renal fibrosis, mesangial expansion, glomerular hypertrophy, oxidative stress and tubular inflammation [36]. Given the expression of ICAM-1 on glomerular endothelium and that of VCAM-1 on glomerular parietal epithelium and proximal tubule, it is not surprising that the levels of these adhesion molecules are raised in diabetic nephropathy: a renal microvascular complication of diabetes mellitus in which tubular and glomerular lesions are prominent [37].

Finally, there is still a paucity of information on the role of other adhesion molecules (such as ICAM-2, P-selectin or ligands for L-selectin) in nephropathies despite these recent reports. Apparently, enhanced expression of adhesion molecules occurs in diseases which are usually not associated with leukocytic infiltration. This may imply that leukocyte recruitment is not singly modulated by the profile of adhesion molecules on resident renal cells but also by the nexus between chemo-attractants and other activation signals within the immediate inflammatory environment [13].

\section{Diagnostic applications as biomarkers}

Biomarkers are increasingly becoming dependable tools for improving diagnosis and prognostication in nephrology as they make for more accurate and earlier identification of renal pathology [38]. Among other characteristic features, an ideal biomarker should have an early expression following a potentially reversible organ damage and prompt reduction in levels post-organ damage, as well as a rapid and reliable measurement [39]. In their pathogenic role in some nephropathies, certain adhesion molecules appear to have met these criteria as they are highly expressed in these diseases (Table 2).

For instance, in diabetic nephropathy, several novel biomarkers have been identified, and have been classified as glomerular biomarkers, tubular biomarkers, biomarkers of oxidative stress, biomarkers of inflammation and miscellaneous biomarkers [37]. Among the biomarkers of inflammation, chemotactic cytokines such as TNF- $\alpha$, IL-18, and RANTES are reportedly predictors of diabetic nephropathy in diabetic patients. In one report, urinary TNF-a levels were raised in diabetic patients who had increased urine albumin excretion coupled with the significant rise in urinary TNF- $\alpha$ excretion as diabetic nephropathy progressed: underscoring its prospect as a biomarker for predicting this renal microvascular complication [40]. In other studies, urinary and serum IL-18 levels were observed to have correlated positively with albumin excretion rate in diabetics, thus making them predictors of the progression of diabetic nephropathy $[41,42]$. In addition, urinary RANTES excretion rate was noted to be significantly higher in hyperfiltering than in normofiltering type 1 diabetics due to high intraglomerular pressure which contributes to renal inflammation: a pathogenic component of diabetic nephropathy [43]. Based on the findings of the previously mentioned studies [9,33], other adhesion molecules from the immunoglobulin superfamily and 
Table 2. Expression of adhesion molecules in nephropathies and the potential biomarkers

\begin{tabular}{|c|c|c|}
\hline $\begin{array}{l}\text { Expression of adhesion } \\
\text { molecules }\end{array}$ & Nephropathies & $\begin{array}{l}\text { Potential/established } \\
\text { biomarkers }\end{array}$ \\
\hline - ICAM-1 & $\begin{array}{l}\text {-Diabetic nephropathy } \\
\text {-Ig A nephropathy } \\
\text {-Proliferative grades of lupus } \\
\text { nephritis } \\
\text { - Henoch-Schönlein vasculitis }\end{array}$ & -serum ICAM- $1^{\dagger}$ \\
\hline -Normal or $\downarrow$ ICAM-1 & -Minimal change nephropathy & \\
\hline - $\uparrow \mathrm{VCAM}-1$ & $\begin{array}{l}\text {-Ig A nephropathy } \\
\text {-Lupus nephritis (Class II-V) } \\
\text {-Diabetic nephropathy } \\
\text {-Gouty nephropathy } \\
\text {-Minimal change nephropathy } \\
\text {-Membranous nephropathy }\end{array}$ & -serum VCAM- ${ }^{\dagger}$ \\
\hline $\begin{array}{l}\text {-P-selectin } \\
\text {-E-selectin }\end{array}$ & $\begin{array}{l}\text {-Diabetic nephropathy } \\
\text {-ditto }\end{array}$ & $\begin{array}{l}\text {-serum P-selectin \& } \\
\text { E-selectin }\end{array}$ \\
\hline $\begin{array}{l}\text {-RANTES } \\
\text {-IL-18 }\end{array}$ & $\begin{array}{l}\text {-Diabetic nephropathy } \\
\text {-ditto }\end{array}$ & $\begin{array}{l}\text {-serum \& urinary IL-18 } \\
\text {-urinary RANTES }\end{array}$ \\
\hline
\end{tabular}

ICAM-1: Intercellular adhesion molecule-1; VCAM-1: Vascular cell adhesion molecule-1; IL-18: Interleukin-18; RANTES: Regulated on activation, normal T cell expressed and secreted

${ }^{\dagger}$ Potential/established biomarker in diabetic nephropathy; ${ }^{\uparrow}$ Increased expression; $\downarrow$ Decreased expression

the selectins (serum ICAM-1, VCAM-1, E-selectin and P-selectin levels) can also serve as potential predictors of diabetic nephropathy, having been observed to be significantly elevated in affected patients when compared to their normal controls. Similarly, ICAM-1 may also be useful as a diagnostic biomarker for other nephropathies like active crescentic glomerulonephritis, mesangioproliferative glomerulonephritis, Ig A nephropathy, and proliferative grades of lupus nephritis. Nevertheless, these potential novel biomarkers still require validation before their routine clinical use.

\section{Conclusion}

Adhesion molecules play a critical role in the pathogenesis of several forms of nephropathies. Given their increased expression in native renal cells in these diseases, they can potentially serve as diagnostic biomarker. Whereas ICAM-1 and VCAM-1 are well reported as predictors of some nephropathies, the role of selectins in these diseases is still poorly documented, even though recent studies indicate their proven utility as biomarkers in diabetic nephropathy.

\section{Acknowledgment}

The author wishes to acknowledge the invaluable information obtained from the article in reference 1 during the preparation of the manuscript.

\section{References}

1. Dal Canton A (1995) Adhesion molecules in renal disease. Kidney Int 48: 1687-1696. [Crossref]

2. Miller MD, Krangel MS (1992) Biology and biochemistry of the chemokines: A family of chemotactic and inflammatory cytokines. Crit Rev Immunol 12: 17-46. [Crossref]

3. Springer TA (1994) Traffic signals for lymphocyte recirculation and leukocyte emigration: The multistep paradigm. Cell 76: 301-314.

4. Ruoslahti E, Pierschbacher MD (1987) New perspectives in cell adhesion: RGD and integrins. Science 238: 491-497. [Crossref]

5. Springer TA (1990) Adhesion receptors of the immune system. Nature 346: 425-434. [Crossref]
6. Ruoslahti E (1991) Integrins. J Clin Invest 87: 1-5. [Crossref]

7. Couser WG (1990) Mediation of immune glomerular injury. J Am Soc Nephrol 1: 1329. [Crossref]

8. Hora K, Satriano JA, Santiago A (1992) Receptors for IgG complexes activate the synthesis of monocyte chemoattractant peptide-1 and colony-stimulating factor-1. Proc Natl Acad Sci USA 89: 1745-1749.

9. Avci E, Uzeli S (2016) The role of adhesion molecules and cytokines in patients with diabetic nephropathy. Biomed Res: S343-S348.

10. Hemler ME (1990) VLA proteins in the integrin family: Structures, functions, and their role on leukocytes. Annu Rev Immunol 8: 365-400. [Crossref]

11. Ginsberg MH, Loftus JC, Plow EF (1988) Cytoadhesins, integrins, and platelets. Thromb Haemost 59: 1-6. [Crossref]

12. Dejana E, Raiteri M, Resnati M, Lampugnani MG (1993) Endothelial integrins and their role in maintaining the integrity of the vessel wall. Kidney Int 43: 61-65. [Crossref]

13. Brady HR (1994) Leukocyte adhesion molecules and kidney diseases. Kidney Int 45: 1285-1300. [Crossref]

14. Bevilacqua MP (1993) Endothelial-leukocyte adhesion molecules. Annu Rev Immunol 11: 767-804. [Crossref]

15. Bevilacqua MP, Stengelin S, Gimbrone MA, Seed B (1989) Endothelial leukocyte adhesion molecule 1: An inducible receptor for neutrophils related to complement regulatory proteins and lectins. Science 243: 1160-1165.

16. Mcever RP, Beckstead JH, Moore KL, Marshali-Carlson L, Bainton DF (1989) GMP-140, a platelet alpha-granule membrane protein, is also synthesized by vascular endothelial cells and is localized in Weibel-Palade bodies. J Clin Invest 84: 92-99.

17. Patel KD, Zimmerman GA, Prescott SM, McEver RP, McIntyre TM (1991) Oxygen radicals induce human endothelial cells to express GMP-140 and bind neutrophils. $J$ Cell Biol 112: 749-759. [Crossref]

18. Springer TA, Lasky LA (1991) Cell adhesion. Sticky sugars for selectins. Nature 349 196-197. [Crossref]

19. Pober JS, Gimbrone MA, Lapierre LA (1986) Overlapping patterns of activation of human endothelial cells by interleukin-1, tumor necrosis factor, and immune interferon. J Immunol 137: 1893-1896.

20. McLean A (1994) Regulation with RANTES. Lancet 343: 189-190. [Crossref]

21. Kawasaki K, Yaoita E, Yamamoto T, Tamatani T, Miyasaki M, et al. (1993) Antibodies against intercellular adhesion molecule-1 and lymphocyte function-associated antigen-1 prevent glomerular injury in rat experimental crescentic glomerulonephritis. J Immunol 150: 1074-1083.

22. Muller GA, Markovic-Lipkovski J, Muller CA (1991) Intercellular adhesion molecule-1 expression in human kidneys with glomerulonephritis. Clin Nephrol 36: 203-208.

23. Lhotta K, Neumayer HP, Joannidis M, Geissler D, Konig P (1991) Renal expression of intercellular adhesion molecule-1 in different forms of glomerulonephritis. Clin Sci 81: $477-481$.

24. Fuiano G, Sepe V, Ferrone S (1990) Expression of intercellular adhesion molecule-1 in necrotizing glomerulonephritis. J Am Soc Nephrol 1: 560.

25. Waldherr R, Eberlein-Gonska M, Noronha IL, Andrassy K, Ritz E (1990) TNF-a and ICAM-1 expression in renal disease. J Am Soc Nephrol 1: 544.

26. Dal Canton A, Fuiano G, Sepe V, Caglioti A, Ferrone S (1992) Mesangial expression of intercellular adhesion molecule-1 in primary glomerulosclerosis. Kidney Int 41: 951955

27. Markovic-Lipkovski J, Muller CA, Risler T, Bohle A, Muller GA (1991) Mononuclear leukocytes, expression of HLA class II antigens and intercellular adhesion molecule-1 in focal segmental glomerulosclerosis. Nephron 58: 286-293.

28. Rothlein R, Mainolfi EA, Czajkowski M, Marlin SD (1991) A form of circulating ICAM-1 in human serum. J Immunol 147: 3788-3793. [Crossref]

29. Seron D, Cameron JS, Haskard DO (1991) Expression of VCAM-1 in the normal and diseased kidney. Nephrol Dial Transplant 6: 917-922. [Crossref]

30. Wutrich RP (1992) Vascular cell adhesion molecule-1 (VCAM-1) expression in murine lupus nephritis. Kidney Int 42: 903-914. [Crossref]

31. Wutrich RP, Jevnikar AM, Takei F, Glimcher LH, Kelley VE (1990) Intercellular adhesion molecule-i expression is upregulated in auto-immune murine lupus nephritis. Am J Pathol 136: 441-450. 
32. Yokoyama H, Tomosugi N, Takaeda M (1992) Glomerular expression of cellular adhesion molecules and serum TNF-alpha and soluble ICAM-1 levels in human glomerulonephritis. J Am Soc Nephrol 3: 669.

33. Luis-Rodriguez D, Martinez-Castelao A, Gorriz JL, De-Alvaro F, Navarro-Gonzalez JF (2012) Pathophysiological role and therapeutic implications of inflammation in diabetic nephropathy. World J Diabetes 15: 7-18.

34. Ted Wu, Kristine CY McGrath, Alison K Death (2005) Cardiovascular disease in diabetic nephropathy patients: Cell adhesion molecules as potential markers. Vasc Health Risk Manag 1: 309-316.

35. Tavridou A, Georgoulidou A, Roumeliotis A (2015) Association of plasma adiponectin and oxidized low-density lipoprotein with carotid intima-media thickness in diabetic nephropathy. J Diabetes Res 1: 507265. [Crossref]

36. Arora MK, Singh UK (2013) Molecular mechanisms in the pathogenesis of diabetic nephropathy: An update. Vascul Pharmacol 58: 259-271.

37. Uwaezuoke SN (2017) The role of novel biomarkers in predicting diabetic nephropathy: A review. Int $J$ Nephrol Renovasc Dis 10: 221-231.
38. Mischak H, Delles C, Vlahou A, Vanholder R (2015) Proteomic biomarkers in kidney disease: Issues in development and implementation. Nat Rev Nephrol 11: 221-232. [Crossref]

39. Cruz DN, Goh CY, Haase-Fielitz A, Ronco C, Haase M (2010) Early biomarkers of renal injury. Congest Heart Fail 16: 25-31. [Crossref]

40. Navarro JF, Mora C (2006) Diabetes, inflammation, proinflammatory cytokines, and diabetic nephropathy. Scientific World Journal 6: 908-917.

41. Nakamura A, Shikata K, Hiramatsu M (2005) Serum interleukin-18 levels are associated with nephropathy and atherosclerosis in Japanese patients with type 2 diabetes. Diabetes Care 28: 2890-2895.

42. Kajitani N, Shikata K, Nakamura A, Nakatou T, Hiramatsu M, et al. (2010) Microinflammation is a common risk factor for progression of nephropathy and atherosclerosis in Japanese patients with type 2 diabetes. Diabetes Res Clin Pract 88: 171-176. [Crossref]

43. Har R, Scholey JW, Daneman D (2013) The effect of renal hyperfiltration on urinary inflammatory cytokines/chemokines in patients with uncomplicated type 1 diabetes mellitus. Diabetologia 56: 1166-1173.

Copyright: (O2019 Uwaezuoke SN. This is an open-access article distributed under the terms of the Creative Commons Attribution License, which permits unrestricted use, distribution, and reproduction in any medium, provided the original author and source are credited. 